\section{GET THEM BRUSHING!}

The Aquafresh Kid's Motivation Pack is a great way to help instil good dental care habits in your younger patients - habits which could last them a lifetime. The kits are bursting with colourful materials, all aimed at making brushing fun and visits to the dentist less intimidating.

Inside you'll find 'well done' stickers to reward dental attendance, 'Nurdle' passports for parents to record children's dental visits, brushing charts, and other activities for your young patients and their parents. For your surgery there's a poster and a display card - to let everyone know that yours is a 'child-friendly practice'.

The free packs can be ordered at www.gskdentalprofessionals.co.uk.

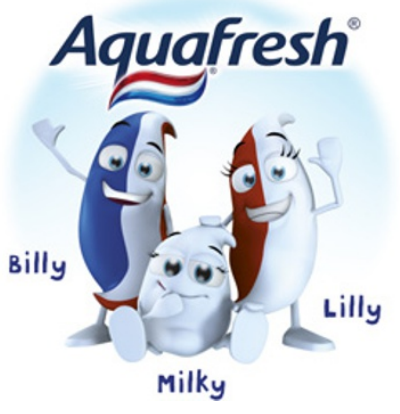

\section{EUROPE UNITES FOR A SMILE}

Wrigley and GlaxoSmithKline Consumer Healthcare are proud to announce their joint support for the upcoming Smiling Europe Campaign which will raise public awareness of oral diseases and promote ways to help improve oral health standards in Europe. The Smiling Europe Campaign will start later this year and will feature oral health forums and discussions with dental experts, as well as public awareness raising activities in several European countries.

'I am excited about the launch of this new initiative to raise awareness of oral diseases and to encourage preventive measures to maintain good oral health', said Professor Kenneth Eaton, Chair of the Platform for Better Oral Health in Europe, at the British Dental Conference and Exhibition 2012 in Manchester.

Oral diseases remain a major health challenge across Europe. Most oral diseases are easily preventable by routine hygiene practices, but many Europeans are not aware of these beneficial and preventative practices leading to the need for costly palliative treatment.

www.oralhealthplatform.eu.

\section{CLINICALLY PROVEN TO ELIMINATE ODOUR AND WHITEN TEETH}

UltraDEX (previously known as RetarDEX) with $\mathrm{iQ}^{+}$ActiveOxi Technology is clinically proven to eliminate odour causing volatile sulphur compounds (VSC) on contact and breaks down bacterial biofilm, leading to improved gum and oral health. The $\mathrm{iQ}^{+}$ ActiveOxi Technology combines CloSYS II (stabilised chlorine dioxide) with sodium monofluorophosphate to work with the natural oral $\mathrm{pH}$. The active ingredient is antimicrobial, killing both aerobic and anaerobic bacteria associated with plaque, tooth decay and biofilm.

Independent clinical tests have proven that the active ingredient in UltraDEX eliminates odour-causing VSCs for fresh breath and UltraDEX is clinically proven to whiten teeth by gently oxidising organic molecules responsible for staining, rather than harsh abrasives, within 14 days.

Three exciting new products have joined the already highly successful UltraDEX range: a Recalcifying \& Whitening Toothpaste, Wire-Free Interdental Brushes and an Interdental Tape both coated with active stabilised chlorine dioxide.

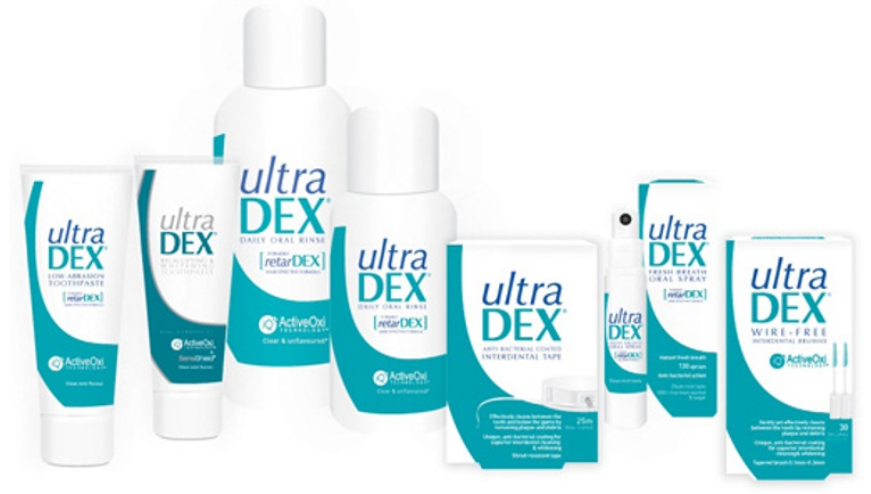

\section{THINK BEYOND PAIN RELIEF}

Sensodyne Repair \& Protect lets you harness advanced bone regeneration technology to help treat and prevent your patients' dentine hypersensitivity. Sensodyne Repair \& Protect is the first daily use toothpaste to combine advanced NovaMin technology with the remineralising ability of fluoride.

NovaMin (calcium sodium phosphosilicate) builds a substantive hydroxyapatite-like layer, not only within dentine tubules but also over exposed dentine to protect against sensitivity. When brushing with Sensodyne Repair \& Protect, NovaMin releases calcium and phosphate ions into saliva and the salivary $\mathrm{pH}$ is elevated to produce favourable conditions for layer formation.

The layer generated is around $50 \%$ harder than natural dentine, and it binds firmly to the dentinal collagen to withstand the daily oral challenges your patients face. The layer begins building from the first use and, with twice-daily brushing, delivers ongoing, clinically proven relief from the pain of dentine hypersensitivity.

Request samples of Sensodyne Repair \& Protect at www.gsk-dentalprofessionals.co.uk and let your patients experience this advanced toothpaste for themselves.

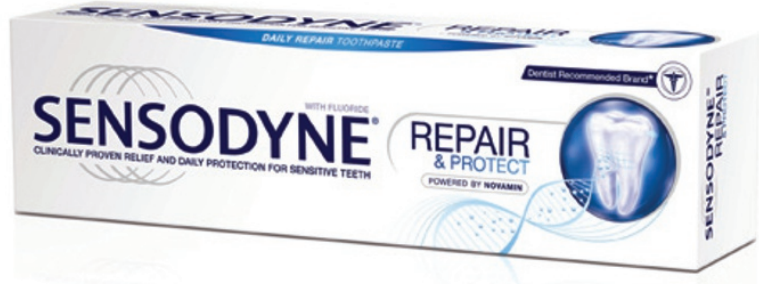

If you would like to promote your products or services direct to the dental industry through VITAL UPDATE telephone Andy on 02078434785. 